\title{
Type 2 diabetes mellitus is associated with increased axial bone density in men and women from the Hertfordshire Cohort Study: evidence for an indirect effect of insulin resistance?
}

\author{
E. M. Dennison ${ }^{1}$ H. E. Syddall ${ }^{1}$ A. Aihie $\operatorname{Sayer}^{1}$ - S. Craighead ${ }^{1}$ D. I. W. Phillips ${ }^{1}$ C. Cooper $^{1}$ \\ ${ }^{1}$ MRC Epidemiology Resource Centre, University of Southampton, Southampton General Hospital, Southampton, UK
}

\begin{abstract}
Aims/hypothesis. Previous studies have suggested that the high bone density often observed in type 2 diabetic patients may be explained by insulin resistance. We explored this hypothesis in the Hertfordshire Cohort Study.

Methods. A total of 465 men and 444 women aged 59 to 71 years and with no prior diagnosis of diabetes attended a clinic where a glucose tolerance test was performed and bone density measured at the femoral neck and lumbar spine. Biochemical markers of bone turnover (serum osteocalcin and urinary mean c-terminal cross-linking telopeptide of type II collagen) were measured in 163 men.

Results. According to WHO criteria, 83 men and 134 women were diagnosed with impaired glucose tolerance and a further 33 men and 32 women were
\end{abstract}

diagnosed as having type 2 diabetes. Bone density was higher in newly diagnosed diabetic subjects, with relationships stronger in women $(p<0.001)$ than men $(p<0.05)$ and attenuated by adjustment for body mass index. In both sexes, we observed positive correlations between the total femur and femoral neck bone mineral density with measures of insulin resistance $(r=0.17-0.22)$, with stronger results observed in women. These relationships did not apply after adjustment for body mass index. Glucose status did not lead to differences in osteocalcin level or c-terminal cross-linking telopeptide of type II collagen levels.

Conclusions/interpretation. Our findings suggest that hyperinsulinaemia may affect bone mineral density through indirect effects, e.g. body weight.

Keywords Bone density $\cdot$ Diabetes $\cdot$ Insulin
Received: 1 March 2004 / Accepted: 10 June 2004

Published online: 24 November 2004

(C) Springer-Verlag 2004

E. M. Dennison (๘)

MRC Epidemiology Resource Centre,

University of Southampton,

Southampton General Hospital,

Southampton, SO16 6YD, UK

E-mail: emd@mrc.soton.ac.uk

Tel.: +44-23-80777624, Fax: +44-23-80704021

Abbreviations: BMD, bone mineral density .

HOMA B, homeostasis model of insulin secretion .

HOMA R, homeostasis model of insulin resistance

\section{Introduction}

While studies of bone density in diabetic patients have given conflicting results, type 2 diabetes is usually associated with increased axial bone density $[1,2,3,4$, $5,6]$, and it has been suggested that this association is due to insulin resistance. A recent cross-sectional study of over 7500 Canadian men and women showed that type 2 diabetes was associated with higher bone mineral density (BMD) even after adjustment for confounding variables, although relationships were stronger in women [7]. The authors speculated that these findings might be due to an anabolic effect of insulin on bone tissue, as type 2 diabetes may be preceded by a period of insulin resistance leading to hyperinsulinaemia. However, they were unable to test these hypotheses. 
Here we present data from the Hertfordshire Cohort Study, in which men and women born in Hertfordshire in the $1930 \mathrm{~s}$ and still resident there were characterised with regard to glucose metabolism, measures of insulin secretion and resistance, and bone mass.

\section{Subjects and methods}

Study protocol and procedures. The selection procedure for the study subjects was as follows. In brief, with the help of the National Health Service Central Registry at Southport, and Hertfordshire Family Health Service Association, we traced men and women who were born in Hertfordshire between 1931 and 1939 and were still living there during the period 1998-2003. Altogether we identified 1760 men and 1447 women. After obtaining written permission from each subject's general practitioner, we approached each person by letter, asking them if they would be willing to be contacted by one of our research nurses. Consent to a home visit by a research nurse was given by 768 men and 714 women. During the visit, subjects filled in a structured questionnaire. This included information on socioeconomic status, medical history, cigarette smoking, alcohol consumption and reproductive variables in women. Physical activity was assessed by a previously validated questionnaire [8]. In brief, a standardised score ranging from 0 to 100 for physical activity was derived from frequency of gardening, housework, climbing stairs and carrying loads in a typical week. Higher scores indicate greater levels of activity. After the nurse's visit, the subject was invited to attend a local clinic. This invitation was taken up by 737 (96\%) men and 675 (95\%) women.

At the clinic, height was measured to the nearest $0.1 \mathrm{~cm}$ using a Harpenden pocket stadiometer (Chasmors, London, UK) and weight to the nearest $0.1 \mathrm{~kg}$ on a SECA floor scale (Chasmors). Body mass index was calculated as weight divided by height $^{2}\left(\mathrm{~kg} / \mathrm{m}^{2}\right)$.

Subjects previously undiagnosed with diabetes (687 men and 644 women) attended the morning clinic after an overnight fast. At this visit, an OGTT was performed using the equivalent of $75 \mathrm{~g}$ anhydrous glucose, with blood samples obtained at baseline, and at 30 and 120 minutes. Plasma insulin and proinsulin levels were measured from the samples obtained. Glucose tolerance was classified on the basis of 2-h glucose for 679 men and 631 women. Subjects who had previously been diagnosed with diabetes (50 men and 31 women) attended the clinic non-fasted, did not participate in the OGTT, and were excluded from subsequent analyses presented in this article.

At the clinic subjects were asked to consent to measurement of BMD by dual energy X-ray absorptiometry at the lumbar spine and proximal femur (neck, total, intertrochanteric and trochanteric regions, Ward's triangle) using a Hologic QDR 4500 instrument (Vertec Scientific, Reading, UK). Individuals taking drugs known to alter bone metabolism (such as bisphosphonates) were excluded from this part of the study, although women taking hormone replacement therapy were allowed to participate. There were no other exclusion criteria to this part of the study. Of the 679 men and 631 women with classified glucose tolerance, $465(68 \%)$ of the men and 444 $(70 \%)$ of the women agreed to measurement of bone density. Measurement precision error, expressed as coefficient of variation, was $1.55 \%$ for lumbar spine BMD, $1.45 \%$ for total femur and $1.83 \%$ for femoral neck BMD for the Hologic QDR 4500; these figures were obtained from 25 volunteers, who were not part of the study, but who underwent two scans on the same day, getting on and off the table between examinations. Shortterm (2-month) precision error for the QDR 4500 was less than $1 \%$ for both sites (manufacturer's figures).

Ethical permission for the study was granted by the East and North Hertfordshire Ethical Committees. All participants gave written informed consent. The study was carried out in accordance with the Declaration of Helsinki as revised in 2000 .

Laboratory measurements. Intact insulin, proinsulin and 32-33 split proinsulin were measured by in-house immunofluorimetric two-site assays (DELFIA system) based on published methods [9]. Inter-assay imprecision ranged from 6 to $10 \%$ (CV\%) for low, medium and high-quality control samples for intact insulin, and from 7 to $15 \%$ for insulin precursors. Glucose was assayed on an Advia 1650 autoanalyser (Bayer Diagnostics, Newbury, UK). The coefficient of variation for this system was $1.1 \%$ at a glucose level of $2.86 \mathrm{mmol} / 1$ and $0.9 \%$ at a glucose level of $16.46 \mathrm{mmol} / \mathrm{l}$.

Diabetes mellitus and impaired glucose tolerance were classified using WHO criteria, i.e. 2-h glucose concentrations of $11.1 \mathrm{mmol} / \mathrm{l}$ or higher and 7.8 to $11.0 \mathrm{mmol} / 1$ respectively [10]. Insulin resistance was assessed according to the homeostasis assessment model of Matthews. First described in 1985, this mathematical model, validated against the gold standard the euglycaemic clamp, is often used in large epidemiological studies [11]. This method yields estimates of insulin resistance, known as the homeostasis model of insulin resistance (HOMA-R) and of insulin secretion, known as the homeostasis model of insulin secretion (HOMA-B).

Serum osteocalcin was measured using an automatic system (Kryptor-Osteo, CisBiointernational, Gif/Yvette, France). The intra-assay coefficient of variation was $<2.5 \%$; the interassay coefficient of variation was $<3 \%$. An overnight urine sample was also obtained; type II collagen cross-linked C-telopeptide was measured using an enzyme-linked immunosorbent assay. The intra-assay coefficient of variation was $<8 \%$ and interassay coefficient of variation was $<10 \%$.

Statistical analysis. Body mass index, HOMA-R and HOMA$\mathrm{B}$ were $\log _{\mathrm{e}}$ transformed to normal distributions. Variables were summarised using means and standard deviations (geometric where appropriate) and tabulations of frequency and percentage distributions. Analysis of variance was used to assess differences in mean BMD according to glucose status (normal, IGT, newly diagnosed diabetes). Pearson correlations were used to analyse the relation between insulin resistance and secretion and BMD. We used multiple linear regression models to adjust for anthropometric and lifestyle factors. All analyses were carried out using STATA, release 7.0. A $p$ value of less than 0.05 was considered statistically significant.

\section{Results}

The characteristics of the study population at baseline are displayed in Table 1. The mean age of the men and women studied was 64.8 and 66.4 years respectively. Of the men $34 \%$ had never smoked (women 63\%), while $52 \%$ of the men ( $28 \%$ of the women) were exsmokers and $14 \%$ of the men (9\% of the women) were current smokers. Four percent of men and 18 percent of women were non-drinkers, while $22 \%$ of men and $12 \%$ of women were moderate drinkers (11-21 and 8-14 units per week respectively, 1 unit being a small 
Table 1. Characteristics of study participants (summary)

\begin{tabular}{|c|c|c|}
\hline Characteristic & Men $(n=465)$ & Women $(n=444)$ \\
\hline Age (years) & $64.8(2.6)$ & $66.4(2.6)$ \\
\hline $\operatorname{BMI}\left(\mathrm{kg} / \mathrm{m}^{2}\right)$ a & $26.6(1.1)$ & $26.8(1.2)$ \\
\hline Habitual activity $(\%)^{\mathrm{c}}$ & $64.3(14.9)$ & $61.5(14.8)$ \\
\hline Current manual social class (IIIM-V) d $n(\%)$ & $181(38.9)$ & $174(39.2)$ \\
\hline Current non-manual social class (I-IIIN) d $n(\%)$ & $258(55.5)$ & $270(60.8)$ \\
\hline Total femoral BMD $\left(\mathrm{g} / \mathrm{cm}^{2}\right)$ e & $1.04(0.13)$ & $0.89(0.13)$ \\
\hline Impaired glucose tolerance $(7.8-11.0 \mathrm{mmol} / \mathrm{l}) n(\%)$ & $83(17.9)$ & $134(30.2)$ \\
\hline Type 2 diabetes $(\geq 11.1 \mathrm{mmol} / \mathrm{l}) n(\%)$ & $33(7.1)$ & $32(7.2)$ \\
\hline HOMA-R * & $3.5(2.0)$ & $3.9(1.9)$ \\
\hline HOMA-B * & $116.7(1.8)$ & $138.4(1.7)$ \\
\hline
\end{tabular}

Values are means (SD) unless stated otherwise. a Geometric mean and SD. b Median and interquartile ranges among drinkers. 18 men and 78 women stated that they do not drink alcohol. 1 unit of alcohol is a small glass of wine or a single measure of spirit ( $8 \mathrm{~g}$ pure alcohol). ${ }^{\mathrm{c}}$ Standardised score ranging 0-100 derived from frequency of gardening, housework, climbing stairs and carrying loads in a typical week. Higher scores indicate greater level of activity. ${ }^{\mathrm{d}}$ Social class was unclassified for 26 men. I-IIIN and IIIM-V denote classes one to three (non-manual), and three (manual) to five, of the 1990 OPCS Standard Occupational Classification scheme for occupation and social class. Social class was identified on the basis of own current or most recent full-time occupation for men and women who had never been married, but on the basis of the husband's occupation for women who were or had been married. e One man was not scanned at the lumbar spine. Three men and one woman were not scanned at the hip

Table 2. Bone mineral density in 465 Hertfordshire men and 444 Hertfordshire women according to 2-h oral glucose tolerance status

\begin{tabular}{|c|c|c|c|c|c|c|}
\hline & \multicolumn{2}{|c|}{ Lumbar spine } & \multicolumn{2}{|c|}{ Femoral neck } & \multicolumn{2}{|l|}{ Total femur } \\
\hline & Men & Women & Men & Women & Men & Women \\
\hline Normal (<7.8 mmol/l) & $1.07(0.16)$ & $0.94(0.18)$ & $0.84(0.11)$ & $0.74(0.11)$ & $1.03(0.13)$ & $0.88(0.13)$ \\
\hline IGT (7.8-11.0 mmol/1) & $1.07(0.17)$ & $0.98(0.15)$ & $0.85(0.14)$ & $0.77(0.12)$ & $1.04(0.15)$ & $0.91(0.14)$ \\
\hline Type 2 diabetes $(\geq 11.1 \mathrm{mmol} / \mathrm{l})$ & $1.16(0.12)$ & $1.07(0.18)$ & $0.90(0.13)$ & $0.83(0.12)$ & $1.11(0.14)$ & $0.97(0.12)$ \\
\hline$p^{c}$ & 0.04 & 0.09 & 0.27 & 0.11 & 0.12 & 0.13 \\
\hline$p^{d}$ & 0.05 & 0.02 & 0.38 & 0.02 & 0.12 & 0.03 \\
\hline$p^{e}$ & 0.05 & 0.07 & 0.25 & 0.08 & 0.08 & 0.08 \\
\hline
\end{tabular}

Bone mineral density (BMD) scores are given as mean (SD); $p$ values are based on 2 degrees of freedom from an ANOVA test for overall difference in BMD according to 2-h glucose tolerance status.

a unadjusted; ${ }^{\mathrm{b}}$ adjusted for BMI; ${ }^{\mathrm{c}}$ adjusted for BMI, age, cigarette and alcohol consumption, current social class, activity

glass of wine or a single measure of spirit). We found that $25 \%$ of men and $3 \%$ of women consumed more than the recommended number of units of alcohol per week ( 22 units or more for men, 15 units or more for women). Standardised physical activity scores were $64 \%$ in men and $62 \%$ in women.

IGT was diagnosed in 83 men and 134 women as a result of this study, and a further 33 men and 32 women were newly diagnosed as diabetic according to our classification. Table 2 shows the mean BMD at each site according to glucose status, with significance lev- level, and in women hormone replacement therapy and menopausal status; d adjusted for body weight; e adjusted for body weight, age, cigarette and alcohol consumption, current social class, activity level, and in women hormone replacement therapy and menopausal status

els unadjusted and adjusted for lifestyle and BMI. Our results demonstrate that in men newly diagnosed with diabetes BMD was significantly higher, but this was not the case for subjects with impaired glucose tolerance (Fig. 1). These relationships were weakened by adjustment for BMI, although results at the lumbar spine remained statistically significant at the 5\% level. In women, a graded relationship was apparent with $\mathrm{BMD}$ rising through impaired glucose tolerance to diabetes (Fig. 1). Again, the relationships were attenuated by adjustment for BMI. 

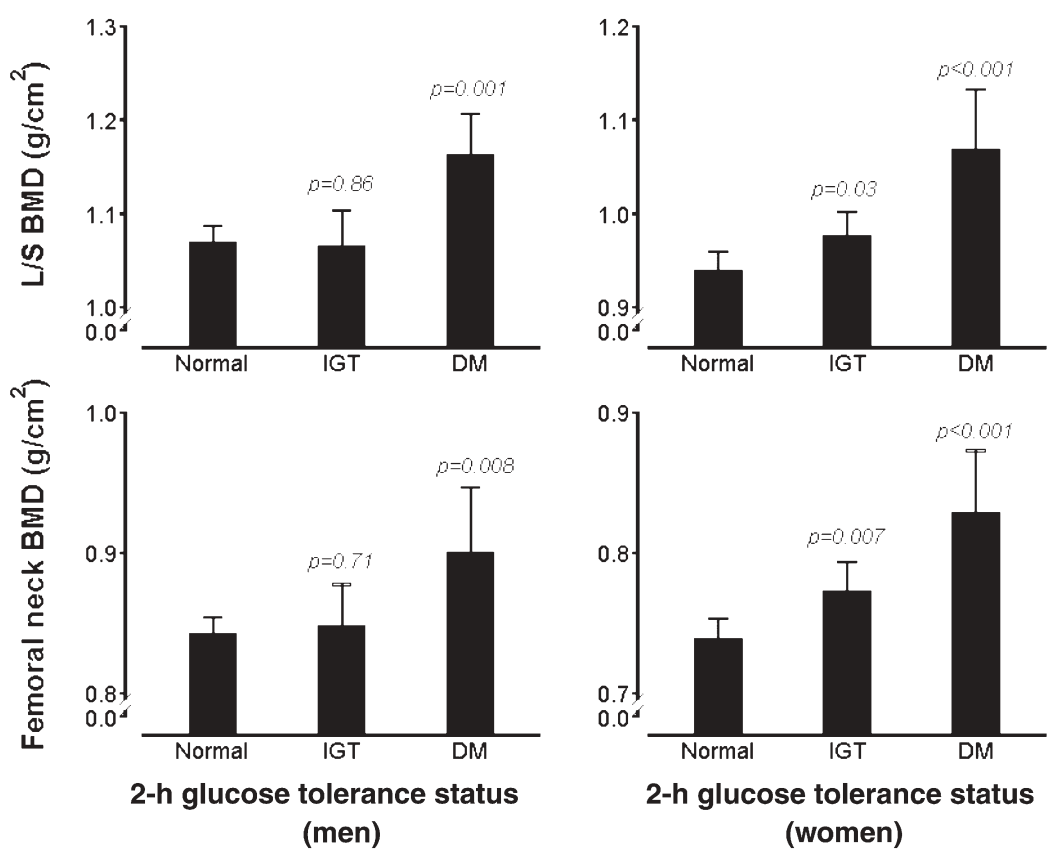

Fig. 1. Relationship between 2-h glucose tolerance status and lumbar spine (L/S) and femoral neck bone mineral density (BMD) in men and women. The $p$ values are for differences in

BMD for IGT vs normal glycaemia, and for type 2 diabetes vs normal glycaemia

Table 3. Bone mineral density (BMD) in relation to insulin resistance and secretion in 465 Hertfordshire men and 444 Hertfordshire women

\begin{tabular}{|c|c|c|c|c|c|c|c|c|}
\hline & \multicolumn{4}{|c|}{ HOMA B (secretion) } & \multicolumn{4}{|c|}{ HOMA R (resistance) } \\
\hline & \multicolumn{2}{|l|}{ Men } & \multicolumn{2}{|l|}{ Women } & \multicolumn{2}{|l|}{ Men } & \multicolumn{2}{|l|}{ Women } \\
\hline Lumbar spine BMD & 0.03 & -0.08 & $0.10^{*}$ & -0.06 & $0.10 *$ & -0.02 & $0.15^{*}$ & -0.05 \\
\hline Femoral neck BMD & 0.11 & -0.05 & $0.11 *$ & $-0.11 *$ & $0.17 * * *$ & -0.01 & $0.17 * * *$ & -0.07 \\
\hline
\end{tabular}

Partial correlations adjusted for $\mathrm{BMI} ; * p<0.05 ; * * * p<0.001$

Table 3 shows the relationships between measures of insulin secretion/resistance and BMD at the different sites, without and with adjustment for BMI. In men, we observed positive correlations between total femur and femoral neck BMD and measures of insulin secretion, although correlations were much stronger between insulin resistance and femoral neck BMD $(r=0.17, p=0.0002)$ and between insulin resistance and total femur BMD $(r=0.18, p=0.0001$, Fig. 2). These relationships did not apply after adjustment for BMI. In women, relationships were seen between BMD at all sites and measures of insulin secretion $(r=0.10-0.17$, Fig. 2) and insulin resistance $(r=0.15-0.22)$. These, too, did not apply after adjustment for BMI. These relationships were little changed by excluding newly diagnosed diabetic subjects; hence total femoral BMD remained significantly correlated with insulin secretion in men $(r=0.12, p<0.05)$ and women $(r=0.17, p=0.001)$ and with insulin resistance in both sexes (men: $r=0.14, p<0.05$; women $r=0.17, p<0.001$ ), although once again results were attenuated by adjustment for BMI.

In view of the high prevalence of spinal osteoarthritis in this age group, we repeated our analyses of relationships with the lumbar spine using only the L1 vertebra, which is often unaffected by osteoarthritis. This made little difference to our results (test for trend of lumbar spine BMD according to diabetic status: men $p=0.008$; women $p=0.02$, fully adjusted).

Measurements of bone turnover were made in a subset of 163 men from this group. We found no significant differences in measurements of osteocalcin or c-terminal cross-linking telopeptide of type II collagen according to glucose tolerance.

\section{Discussion}

We have shown that BMD was higher in middle-aged men and women with newly diagnosed diabetes melli- 

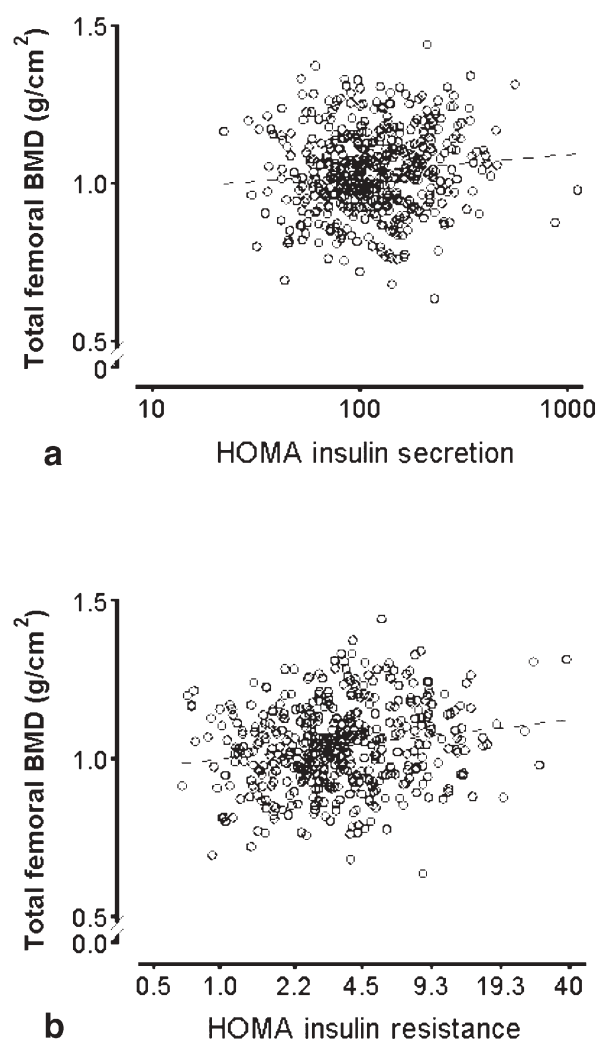

Fig. 2. Relationship between insulin resistance and secretion and total femoral bone mineral density $(\mathrm{BMD})$ in men $(\mathbf{a}, \mathbf{b})$ and women (c, d). HOMA, homeostasis model assessment. $r=0.11 ; p=0.03$ (a); $r=0.18 ; p=0.0001$ (b); $r=0.17 ; p=0.0005$ (c); $r=0.22 ; p=0.0001(\mathbf{d})$

tus than in their normo-glycaemic counterparts, and that, consistent with previous work in this area, the relevant correlations were stronger in women. Although adjustment for adiposity weakened the association, it did not remove it completely. The main strength of this study, and an advance over previous studies $[6,7]$ was the measurement of insulin levels in the cohort; hyperinsulinaemia can affect BMD both directly and indirectly (acting through BMI), and our results would support the latter hypothesis.

Our study has a number of weaknesses. The individuals recruited were selected because they had been born in Hertfordshire, and continued to live there at the age of 59 to 71 years. However, we have previously demonstrated that the Hertfordshire cohort has similar smoking characteristics and bone density to those reported nationally [12], suggesting that selection bias is minimal. Furthermore, we ascertained that those individuals who did not participate in the study had similar birthweights and weights at one year to those who did. We also confirmed that there were no significant differences in anthropometric measures, cigarette or alcohol consumption or physical activity levels between those individuals who did or did not elect to undergo a bone density test. Although measurements of
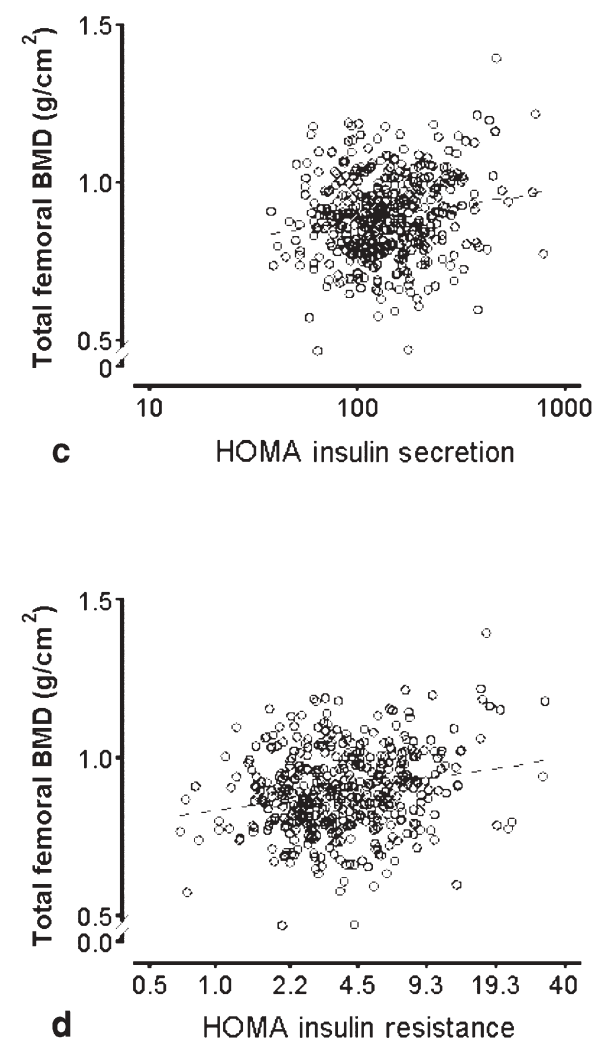

lumbar spine BMD may have been affected by osteoarthritic change, we attempted to remove the effect by looking at relationships between glucose status and the L1 vertebra only, as this bone is often uninvolved in osteoarthritic change. Finally, vitamin D deficiency is a recognised cause of low BMD among older individuals, and although 25-hydroxy vitamin D levels were not measured in this cohort, previous studies of Hertfordshire women who have participated in similar studies have shown vitamin D insufficiency to be uncommon in this group of free-living individuals [13].

Our results suggest that newly diagnosed diabetic subjects who participated in our study had higher BMD than individuals with euglycaemia. Our findings are in accord with the two largest studies on this topic performed to date $[6,7]$, in which men and women with type 2 diabetes had higher BMD after adjustment for BMI and lifestyle factors. The authors of both papers speculated that this might be caused by an anabolic effect of insulin on bone tissue, and certainly we have demonstrated associations between measures of insulin resistance and BMD. Other studies [14, 15] have reported that such associations were much stronger in women with type 2 diabetes than in men. It has also been speculated that hyperinsulinaemia may have a negative impact on sex hormone binding globulin, hence increasing free sex hormone levels and protecting from bone loss; interestingly a large study of 5931 men and women found that men, but not women, with a history of non-vertebral fractures had lower insulin levels 2 hours after a glucose load [16]. Our findings of associations between insulin resis- 
tance and secretion in women would seem to increase the probability of interaction with sex hormones, although we were unable to confirm this in the cohort studied.

Although we have reported higher BMD values in diabetic patients, this may not translate into a lower fracture risk. The Study of Osteoporotic Fractures reported a doubling of intra-articular distal radius fractures among diabetic women [17], in addition to an increased risk of hip and proximal humerus fractures after adjustment for age, BMI and bone density [18]. Other measures of bone quality, namely broad band ultrasound attenuation measured by calcaneal ultrasound, have also been found to be higher in type $2 \mathrm{di}$ abetic women [19]. By contrast, retrospective studies of fracture incidence in diabetic individuals have reported a lower frequency of non-vertebral fractures in women, but not in men [5]. Follow-up of cohorts that include men and assess other aspects of bone quality would hence seem particularly valuable.

The literature on the relationship between biochemical markers of bone turnover and BMD in diabetic and non-diabetic patients suggests that markers of formation and resorption are lower in the diabetic group $[14,20]$, although the study which reported findings in both sexes commented that such differences were apparent in women only [14]. We found no relationship between glucose tolerance and biochemical markers of bone turnover among men in this study, although the numbers studied were small, limiting power.

In conclusion, this study confirms a positive association between BMD and type 2 diabetes, mediated in part through adiposity and more marked in women than men. Furthermore, our work supports a previous hypothesis that insulin resistance may indirectly underlie this association.

Acknowledgements. We thank the men and women who participated in the study and the nurses and radiology staff who administered the bone density measurements. Computing support was provided by Vanessa Cox, and the manuscript was prepared by Gill Strange. Plasma glucose and insulin/ proinsulin were measured by Peter Wood. The study was funded by the Medical Research Council.

\section{References}

1. Leidig-Bruckner G, Ziegler R (2001) Diabetes mellitus a risk for osteoporosis? Exp Clin Endocrinol Diabetes 109 [Suppl 2]:S493-S514

2. Akin O, Gol K, Akturk M, Erkaya S (2003) Evaluation of bone turnover in postmenopausal patients with type 2 diabetes mellitus using biochemical markers and bone mineral density measurements. Gynecol Endocrinol 17:19-29

3. Bauer DC, Browner WS, Cauley JA et al. (1993) Factors associated with appendicular bone mass in older women. The Study of Osteoporotic Fractures Research Group. Ann Intern Med 118:657-665
4. Weinstock RS, Goland RS, Shane E, Clemens TL, Lindsay R, Bilezikian JP (1989) Bone mineral density in women with type II diabetes mellitus. J Bone Miner Res 4:97-101

5. Van Daele PL, Stolk RP, Burger H et al. (1995) Bone density in non-insulin dependent diabetes mellitus. The Rotterdam Study. Ann Intern Med 122:409-414

6. Lunt M, Masaryk P, Scheidt-Nave C et al. (2001) The effects of lifestyle, dietary dairy intake and diabetes on bone density and vertebral deformity prevalence: the EVOS study. Osteoporos Int 12:688-698

7. Hanley DA, Brown JP, Tenenhouse A et al. (2003) Associations among disease conditions, bone mineral density, and prevalent vertebral deformities in men and women 50 years of age and older: cross-sectional results from the Canadian Multicentre Osteoporosis Study. J Bone Miner Res 18:784790

8. Dallosso HM, Morgan K, Bassy EJ, Ebrahim SBJ, Fentem PH, Arie THD (1988) Levels of customary physical activity among the old and very old living at home. J Epidemiol Commun Health 42:121-127

9. Sobey WJ, Beer SF, Carrington CA et al. (1989) Sensitive and specific two-site immunoradiometric assays for human insulin, proinsulin, 65-66 split and 32-33 split proinsulins. Biochem J 260:535-541

10. World Health Organization (1999) Definition, diagnosis and classification of diabetes mellitus and its complications. Report of a WHO consultation, Part I: Diagnosis and Classification of Diabetes Mellitus, Geneva

11. Matthews DR, Hosker JP, Rudenski AS, Naylor BA, Treacher DF, Turner RC (1985) Homeostasis model assessment: insulin resistance and b-cell function from fasting plasma glucose and insulin concentrations in man. Diabetologia 28:412-419

12. Egger P, Duggleby S, Hobbs R, Fall C, Cooper C (1996) Cigarette smoking and bone mineral density in the elderly. J Epidemiol Commun Health 50:47-50

13. Arden NK, Syddall HE, Javaid MK et al. (2004) Early life influences on serum 1,25(0H)2 vitamin D. Paediatr Perinat Epidemiol (in press)

14. El Miedany YM, el Gaafary S, el Baddini MA (1999) Osteoporosis in older adults with non-insulin dependent diabetes mellitus: is it sex related? Clin Exp Rheumatol 17:561-567

15. Barrett-Connor E, Holbrook TL (1992) Sex differences in osteoporosis in older adults with non-insulin dependent diabetes mellitus. JAMA 268:3333-3337

16. Stolk RP, Van Daele PL, Pols HA et al. (1996) Hyperinsulinaemia and bone mineral density in an elderly population: the Rotterdam Study. Bone 18:545-549

17. Vogt MT, Cauley JA, Tomaino MM, Stone K, Williams JR, Herndon JH (2002) Distal radius fractures in older women: a 10 year follow-up study of descriptive characteristics and risk factors. The study of osteoporotic fractures J Am Geriatr Soc 50:97-103

18. Schwartz AV, Sellmeyer DE, Ensrud KE et al. (2001) Older women with diabetes have an increased risk of fracture: a prospective study. J Clin Endocrinol Metab 86:32-38

19. Korpelainen R, Korpelainen J, Heikkinen J, Vaananen K, Keinanen-Kiukaanniemi S (2003) Lifestyle factors are associated with osteoporosis in lean women but not in normal or overweight women: a population-based cohort study of 1222 women. Osteoporosis Int 14:34-43

20. Akin O, Gol K, Akturk M, Erkaya S (2003) Evaluation of bone turnover in post-menopausal patients with type 2 diabetes mellitus using biochemical markers and bone mineral density measurements. Gynecol Endocrinol 17:19-29 